\title{
Uso de pilas de combustible PEM de alta temperatura en una aplicación de cogeneración para aplicaciones de confort
}

\author{
David Quintana, Víctor Sanz i López, Ramon Costa-Castelló \\ Institut de Robòtica i Informàtica Industrial, CSIC-UPC \\ Carles Batlle \\ Departament de Matemàtiques y Institut d'Organització i Control, EPSEVG, UPC
}

\section{Resumen}

Debido a la importancia a escala mundial de la escasez de energía y los efectos del cambio climático están emergiendo alernativas a las fuentes de energía clásicas como los combustibles fósiles y las centrales de energía nuclear de fusión. En este contexto se encuadran las técnicas de "combined heat and power" como una posible solución debido a su capacidad para generar energía eléctrica y térmica de manera más eficiente que los métodos convencionales. Respecto a esto, las pilas de combustible de alta temperatura con membrana de intercambio de protones permiten implementar sistemas de "combined heat and power" además de tratarse de una tecnología eficiente y con menor impacto en la producción de gases de efecto invernadero. En este artículo se describe un sistema de gestión de energía para un modelo simple basado en una pila de combustible usando control predictivo. Para ello se presentan las características de la pila y su contribución a la solución de la problemática energética actual en primer término. Posteriormente, se describe una modelización y control de bajo nivel de la pila y sus subsistemas de generación de calor y electricidad y, finalmente, un problema de control predictivo es propuesto como un método de gestión del calor y energía eléctrica que cumpla una determinada demanda residencial.

Palabras clave: Gestión de energía, Model Predictive Control y pilas de combustible.

\section{INTRODUCCIÓN}

Entre los principales problemas mundiales, la posible escasez energética en los próximos años y las consecuencias del cambio climático representan unos de los más importantes a juzgar por la actitud mostrada por parte de diversas instituciones alrededor del planeta. El consumo de energía primaria ha crecido un $2,5 \%$ recientemente ([1]). Según los mismos estudios, el consumo de carbón ha incluso incrementado hasta un máximo de un $29,9 \%$ de la energía primaria mundial en el año 2012. En el mismo periodo, las secuelas del terremoto y su consecuente tsunami acaecido en 2011 en la central nuclear de Fukushima, en Japón, empujó a dicho gobierno a reducir la dependencia del país nipón de la energía nuclear de fisión en un $89 \%$ (6,9\% mundialmente). Considerando que los niveles de eficiencia de las centrales de carbón pueden alcanzar el $41 \%$ se ha puesto de manifiesto que la reducción de la proporción de energía térmica desperdiciada es una de las metas de cara a un futuro cercano.

Este curso de los acontecimientos está presionando a las autoridades políticas alrededor del mundo para buscar nuevas formas de energía que incluyan una gestión de la energía desperdiciada adecuadamente. Para usos residenciales, el consumo energético se puede clasificar en un $27 \%$ de la energía eléctrica total y un $38 \%$ de la energía térmica alrededor del mundo. Diferentes usos para el consumo residencial pueden definirse en función del país como puede verse en la figura 1 .

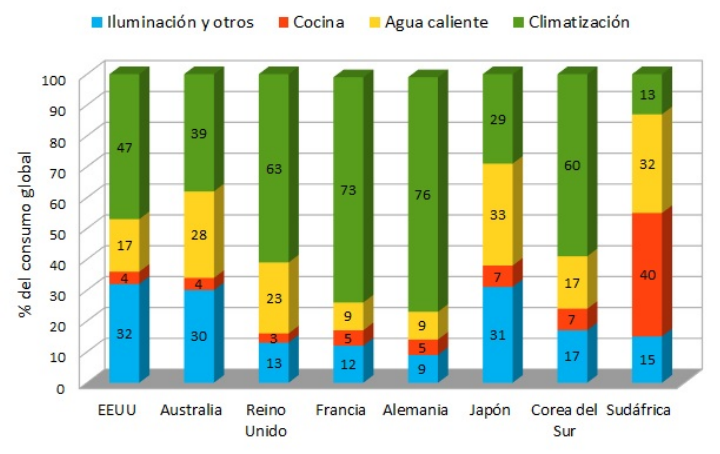

Figura 1: Consumo energético residencial en diferentes países $([1])$

En este contexto, la cogeneración o "combined heat and power" (CHP) ha emergido como una opción para los próximos años debido a su aprovechamiento de ambos tipos de energía, eléctrica y térmica. Entre las diversas tecnologías de CHP, las pilas de combustible son una de las principales opciones propuestas por varios estados como una vía para cambiar parte del sistema de generación energética mundial. Este artículo se centra específicamente en las pilas de combustible de alta temperatura con membrana de intercambio de 
protones (HT-PEMFC) y su utilización para gestión de energía en un sistema de CHP residencial teniendo en cuenta a grandes rasgos sus características físicas y especificaciones técnicas.

En el presente artículo, la caracterización y modelado de una pila HT-PEMFC se presenta, así como los elementos auxiliares para el sistema de CHP como los depósitos de agua para calefacción, agua caliente sanitaria y baterías. Posteriormente se presenta un modelo de control propuesto para cada uno de los elementos por separado para garantizar las especificaciones. Finalmente, se detalla un nivel de control superior con objetivos de gestión de energía y restricciones operacionales.

\section{CARACTERÍSTICAS DE LAS PILAS HT-PEMFC}

Una pila HT-PEMFC ([2]) está compuesta por un ánodo alimentado con hidrógeno puro $\left(\mathrm{H}_{2}\right)$, un cátodo suministrado con oxígeno puro $\left(\mathrm{O}_{2}\right)$ o aire y una membrana que los combina. El hidrógeno se oxida en el ánodo y, en consecuencia, $\mathrm{H}^{+}$(protones) fluyen a través de la membrana polimérica de ánodo a cátodo. Simultáneamente, una corriente de $\mathrm{e}^{-}$(electrones) circula a través de una carga conectada en paralelo a la pila. En el cátodo, oxígeno, electrones y protones se combinan para producir agua (vapor en el caso de alta temperatura) (figura 2) el ánodo. Las reacciones químicas son

$\mathrm{H}_{2} \longrightarrow 2 \mathrm{H}^{+}+2 \mathrm{e}^{-}$,

$\frac{1}{2} \mathrm{O}_{2}+2 \mathrm{e}^{-}+2 \mathrm{H}^{+} \longrightarrow \mathrm{H}_{2} \mathrm{O}$.

Este proceso produce energía eléctrica, calor y agua. El esquema del proceso es el de la figura 2.

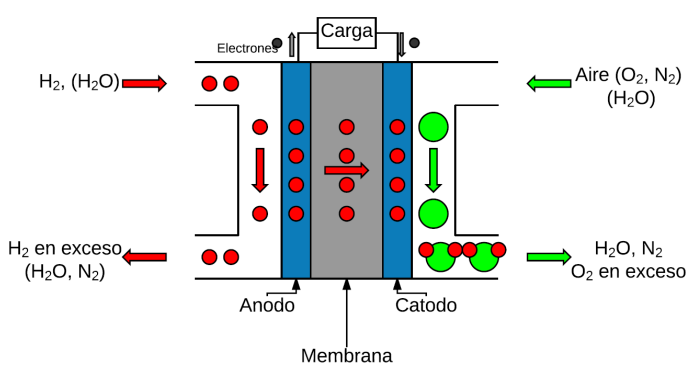

Figura 2: Esquema de la pila

Despreciando las pérdidas del proceso, el potencial reversible de Nernst $E_{N}$ en bornes de la pila se define como:

$$
\begin{aligned}
E^{0} & =-\frac{\Delta g_{f}^{0}}{2 F} \\
E_{N} & =E^{0}+\frac{R T}{2 F} \ln \left(\frac{P_{H_{2}}}{P_{0}}\right)+\ln \left(\frac{P_{O_{2}}}{P_{0}}\right),
\end{aligned}
$$

where

- $\Delta g_{f}^{0}=-228,59 \mathrm{~kJ} / \mathrm{mol}$ es la energía libre de Gibbs para formar un mol de vapor de agua.

- $R=8,31 J /(K \cdot \mathrm{mol})$ es la constante de los gases ideales.

- $T$ es la temperatura.

- $P_{H_{2}}$ es la presión parcial del hidrógeno.

- $P_{\mathrm{O}_{2}}$ es la presión parcial del oxígeno.

- $P_{0}$ es la presión atmosférica.

La tensión eléctrica $V_{\text {celda }}$ de una celda de la pila HT-PEMFC se modeliza típicamente mediante la fórmula de la curva de polarización ([3]) como sigue:

$$
\begin{aligned}
V_{\text {celda }} & =\frac{-\Delta G}{n F}+\frac{R T}{n F} \ln \left(\frac{\left(P_{\mathrm{H}_{2}}\right)^{2}\left(P_{\mathrm{O}_{2}}\right)^{\frac{1}{2}}}{P_{\mathrm{H}_{2} \mathrm{O}}}\right) \\
& -\frac{R T}{\alpha F} \ln \left(\frac{i}{i_{0}}\right)-\frac{R T}{n F} \ln \left(\frac{i_{l}}{i_{l}-i}\right)-i R_{i}
\end{aligned}
$$

Dicha curva de polarización tiene la forma de la figura 3 para los valores de la tabla:

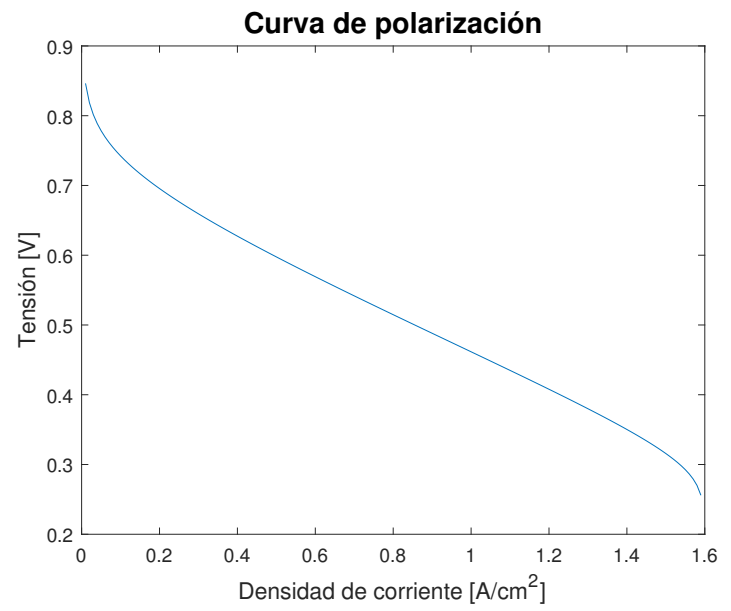

Figura 3: Curva de polarización de la pila HTPEM 


\section{APLICACIÓN A CHP}

El sistema de CHP estudiado es el de la figura 4 y dispone de los siguientes elementos:

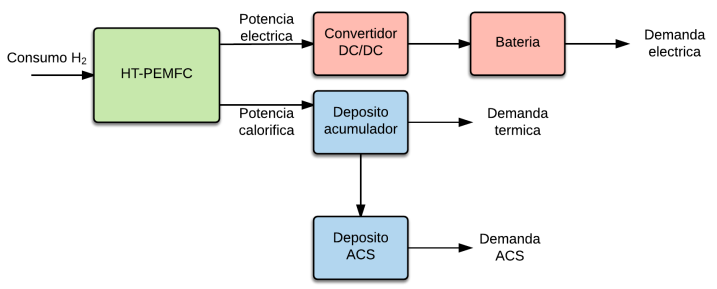

Figura 4: Sistema de CHP

- Pila HT-PEMFC: genera electricidad y calor.

- Convertidor $D C / D C$ : establece el nivel de tensión eléctrica deseado.

- Batería: almacena la energía eléctrica para después suministrarla en función de la demanda.

- Depósito acumulador: almacena agua para generar calor para climatización.

- Depósito $A C S$ : almacena y suministra agua caliente sanitaria (ACS) para uso humano.

El sistema térmico y los respectivos flujos de agua se indican en la figura 5:

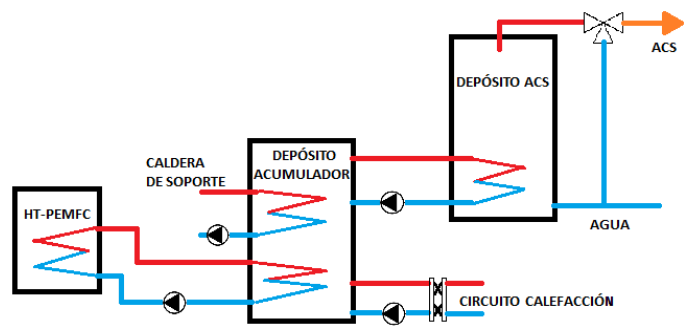

Figura 5: Esquema del sistema térmico

\subsection{PILA HT-PEMFC}

La potencia total teórica generada por la pila $P_{\text {teórica }}$ (si toda la energía química se convirtiera en electricidad) se puede dividir en potencia eléctrica $P_{\text {elec }}$ y potencia calorífica $P_{c a l}$ como sigue (equaciones (4) a (6)):

$$
\begin{aligned}
P_{\text {teórica }} & =V_{t} I N_{\text {celdas }} \\
P_{\text {elec }} & =V I N_{\text {celdas }} \\
P_{\text {cal }} & =P_{\text {teórica }}-P_{\text {elec }} \\
& =I N_{\text {celdas }}\left(V_{t}-V\right)
\end{aligned}
$$

Las curvas de potencia eléctrica $P_{\text {elec }}$ y calorífica $P_{c a l}$ en función de la corriente $I$ se muestran en la figura 6:

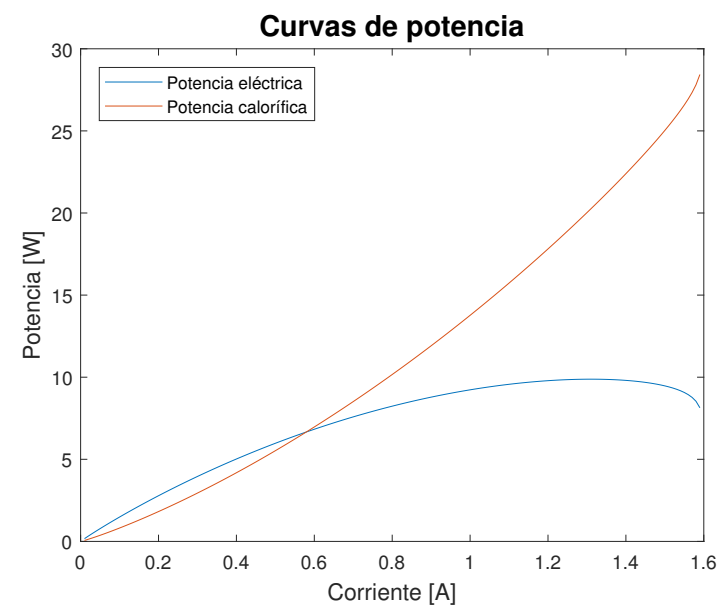

Figura 6: Curvas de potencia eléctrica y calorífica en función de la corriente de la HT-PEMFC

Donde $V_{t}$ es la tensión teórica, $I$ es la corriente de la pila, $N_{\text {celdas }}$ es el número de celdas de la pila y $V$ es la tensión real.

\subsection{DIMENSIONADO EN FUNCIÓN DE LA DEMANDA}

En primer término se asumen valores para la demanda media de una pequeña casa alimentada mediante una pila HT-PEMFC situada en Valencia. En el mes de más consumo (julio, debido al consumo de aire acondicionado). El cálculo de la demanda eléctrica $\hat{P}_{\text {elec }}$ es el siguiente:

$$
\hat{P}_{\text {elec }}=\frac{753 k W h}{m e s} \cdot \frac{1 m e s}{720 h}=1,05 k W
$$

El dimensionado térmico y eléctrico están relacionados a través del rendimiento de la pila. Un rendimiento más elevado significa menos potencia calorífica por cada vatio eléctrico producido. El rendimiento depende del punto de trabajo de la curva de polarización: a más intensidad, menos rendimiento. También es función de la temperatura: a más temperatura, más rendimiento. No obstante, este margen de decisión es relativamente pequeño (el rendimiento siempre oscilará entre el 40 y $60 \%$ ). Por lo tanto, con una pila de $1,5 \mathrm{~kW}$, se podrían extraer alrededor de $1,5 \mathrm{~kW}$ de potencia calorífica. Esta potencia no sería suficiente para alimentar el agua caliente y la calefacción durante los meses de invierno así que se debe pensar en alguna manera de calentar la casa. Existen varias opciones, que se enumeran a continuación:

- Contar con una caldera de apoyo responsable 
de aportar el extra en los meses de diciembre, enero y febrero, que alimentaría el depósito acumulador.

- Trabajar en puntos de rendimiento bajos y dotar el acumulador de agua caliente (fuente del sistema de calefacción) de una resistencia eléctrica. De este modo podría transmitirse la energía eléctrica no tan necesaria en invierno hacia el sistema térmico. Para esta solución probablemente se debería sobredimensionar un poco la pila.

Por lo que respecta a la temperatura del depósito de agua caliente es deseable que sea siempre la establecida, en este caso, de $60{ }^{\circ} \mathrm{C}$. Esto se debe a que el consumo de agua caliente de los hogares acostumbra a estar muy concentrado (sobre todo a primera hora de la mañana debida a las duchas). Al tratarse de un sistema que no es demasiado rápido para hacer frente a imprevistos se debe disponer de un depósito siempre lleno. Además, $60^{\circ} \mathrm{C}$ es la temperatura mínima de acumulación que exige la legislación (Real Decreto 865/2003) para prevenir la legionela. En segundo lugar, se debe mantener un control de la temperatura de la pila ya que, como se ha comentado anteriormente, no funcionará por debajo de $\operatorname{los} 100^{\circ} \mathrm{C}$ y puede degradarse si se alcanzan los $200^{\circ} \mathrm{C}$. Lo ideal es mantenerse en el intervalo $120-180^{\circ} \mathrm{C}$. En tercer lugar, para poder transmitir el calor mediante la convección forzada del serpentín de la ACS desde el depósito acumulador, hace falta que la temperatura de este último sea superior. Con estas restricciones impuestas, se puede jugar con la temperatura del depósito y el nivel de carga (SOC) de la batería.

\section{MODELIZACIÓN Y CONTROL}

\subsection{DEPÓSITO DE ACS}

Cómo se ha visto anteriormente, el depósito de ACS es alimentado por el depósito acumulador a través de un serpentín. La potencia disipada del serpentín hacia el depósito depende de las características físicas del propio serpentín (área de contacto, grueso, material...), de la temperatura del mismo depósito y de la temperatura y caudal del flujo de agua que circula por el serpentín. Estos cálculos de transferencia de calor a menudo se realizan para modelos concretos de forma experimental o por simulación numérica. Se asumirá que el actuador que controla esta potencia es la bomba de agua que hace circular de forma variable el caudal que pasa por el serpentín. Cuando el actuador interrumpe el caudal de agua, no hay transferen- cia de calor. En un cierto rango de funcionalidad, cuanto más caudal circula más potencia se disipa. Este actuador es controlado por un controlador proporcional-integral (PI), la función del cual es mantener la temperatura de la ACS constando a la temperatura deseada, $T_{A C S}^{d e s}$, a $60^{\circ} \mathrm{C}$ mediante un lazo cerrado. El sistema estará sujeto a las perturbaciones $P_{A C S}^{\text {des }}$ (potencia extraída del depósito) y $T_{\text {casa }}$. El controlador elegido ha sido un PI, que asegura un error estacionario nulo ya que, como se verá, la transmitancia de enlace es de orden 1. El lazo superior, que puede considerarse la función de transferencia de la planta (depósito), es de primer orden y tiene la siguiente forma (equación (8)):

$$
G_{p}^{A C S}(s)=\frac{1}{m_{A C S} c_{p, H_{2} O} s+h A_{A C S}}
$$

\subsection{PILA HT-PEMFC}

Para asegurar que la temperatura de trabajo de la pila se sitúe en los puntos de interés, y nunca sobrepasando los límites de 120 y $180^{\circ} \mathrm{C}$, hay que controlar esta variable también mediante un lazo cerrado y un controlador. Cómo en el depósito de ACS, la función del controlador es regular la potencia extraída de la pila a través de un actuador (bomba). Pese a todo, al diseñar el sistema de control de la pila aparece una pequeña dificultad porque el potencial que ofrece depende de forma no lineal de la propia temperatura. Para simplificar el modelo de control se asume un modelo lineal de de la planta de la pila de manera muy similar al del depósito de ACS. La expresión es la que se detalla en la equación (9):

$$
G_{p}^{p i l a}(s)=\frac{1}{m_{\text {pila }} c_{p, p i l a} s+h A_{\text {pila }}}
$$

El controlador usado es también uno de tipo PI. Se debe tener en cuenta problemas debidos a la aparición de mecanismos de "wind-up" y la estructura del esquema de control se debe diseñar adecuadamente para evitarlo.

\section{GESTIÓN DE ENERGÍA}

Para ajustar los valores de calor y electricidad generados a la demanda de una casa tipo se plantea un problema de control predictivo con el objetivo de minimizar el consumo de hidrógeno de la pila HT-PEMFC. El problema tiene, pues, la siguiente forma:

$$
\begin{array}{ll}
\min & \mathbf{f}=\mathbf{u}(k) \\
& \mathbf{x}(k+1)=\mathbf{A x}(k)+\mathbf{B u}(k)+\mathbf{d}(k)
\end{array}
$$


El significado de las variables es:

- $\mathbf{x}(k)=\left[E_{\text {elec }}^{\text {alm }}, E_{\text {cal }}^{\text {alm }}\right]^{T}$ es el vector de estados formado por la energía eléctrica almacenada $E_{\text {elec }}^{a l m}$ y la energía calorífica total almacenada $E_{\text {cal }}^{\text {alm }}$.

- $\mathbf{u}(k)=I(k)$ es el vector de entrada, que corresponde a la corriente de entrada de la pila $I(k) . I(k)$ es proporcional al flujo de hidrógeno.

- $\mathbf{d}(k)=\left[D_{\text {elec }}, D_{\text {cal }}^{A C S}+D_{\text {cal }}^{\text {clim }}\right]^{T}$ es el vector de pertorbaciones compuesto por las demandas previstas en concepto de energía eléctrica $D_{\text {elec }}$ y de energía calorífica para agua caliente sanitaria $D_{c a l}^{A C S}$ para climatización $D_{c a l}^{\text {clim }}$.

- $\mathbf{A}=\mathbf{I}_{2 \times 2}$ es la matriz de estados.

- $\mathbf{B}=\left[E_{\text {elec }}^{\text {prod }}, E_{\text {cal }}^{\text {prod }}\right]^{T}$ es la matriz de entradas, con la energía producida en forma eléctrica $E_{\text {elec }}^{\text {prod }}$ y calorífica $E_{\text {cal }}^{\text {prod }}$.

Los pasos a seguir en el control son los siguientes:

- Definir un horizonte del problema $N_{\text {total }}$ y un horizonte de control $N<N_{\text {total }}$.

- Definir unos valores de demanda prevista para el horizonte $N, \hat{\mathbf{d}}(k) \cdots \hat{\mathbf{d}}(k+N)$.

- Solucionar el problema de optimización para obtener la acción de control $u(k)$ tal que los siguientes $N$ estados predichos $\hat{\mathbf{x}}(k) \cdots \hat{\mathbf{x}}(k+$ $N)$ cumplan las especificaciones.

- Repetir el proceso para cada instante $k$.

Los perfiles de demanda definidos son los consumidos por una casa tipo situada en la costa Mediterránea en un mes de abril. En las figuras 7 y 8 se representan las demandas eléctrica, de calefacción y de agua caliente sanitaria (ACS):

Usando dichos valores como demandas esperadas se ha implementado el problema de optimización descrito en las equaciones (10) y (11) mediante el entorno Yalmip incluido en Matlab. Los resultados obtenidos son los estados $\mathbf{x}(k)=\left[E_{\text {elec }}^{\text {alm }}, E_{\text {cal }}^{a l m}\right]^{T}$ (energía en las unidades de almacenamiento, en las figura 9 y 10) y entradas de control $\mathbf{u}(k)=I(k)$ (corriente de la pila de $N_{\text {celdas }}$, en la figura 11). Estos resultados se representan para un periodo de 8 días tomando muestras cada 15 minutos.

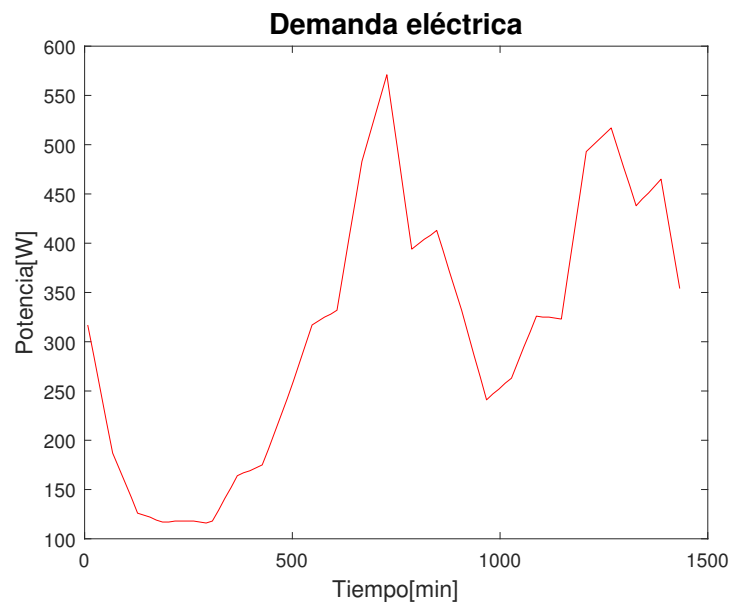

Figura 7: Demanda eléctrica para un día del mes de abril

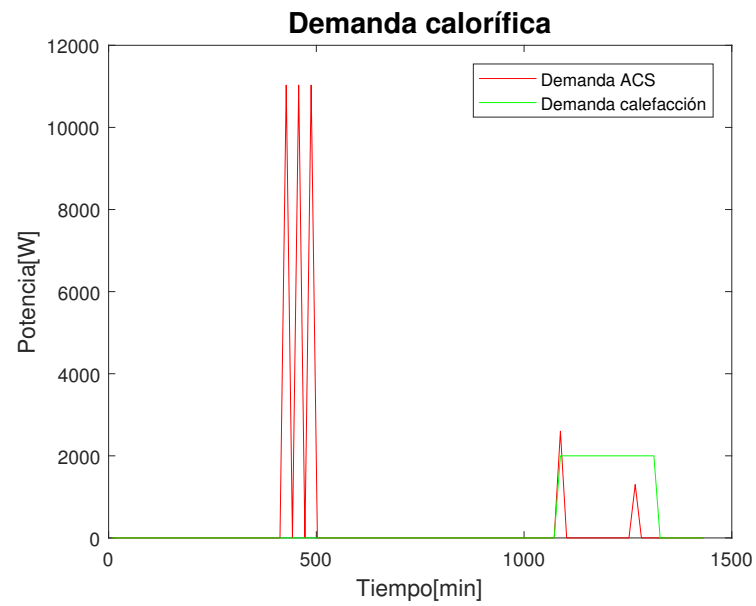

Figura 8: Demanda de calefacción y ACS para un día del mes de abril

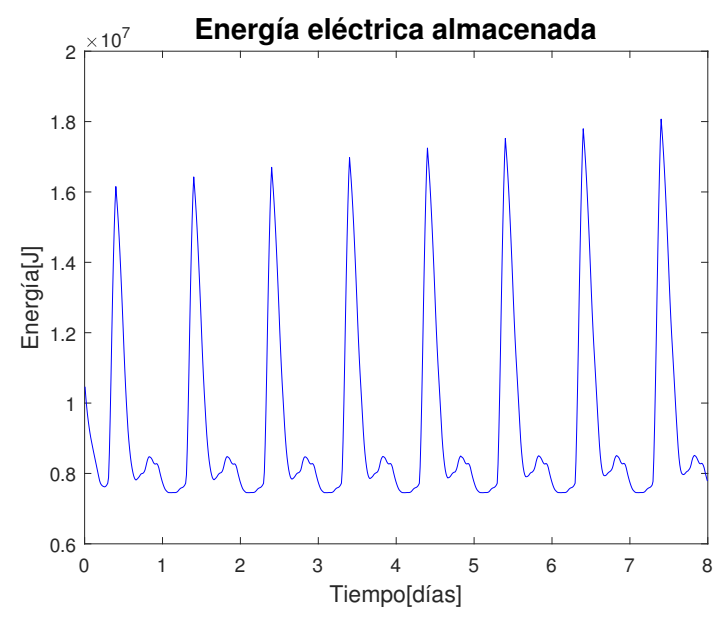

Figura 9: Energía eléctrica almacenada en la batería a lo largo de ocho días del mes de abril 


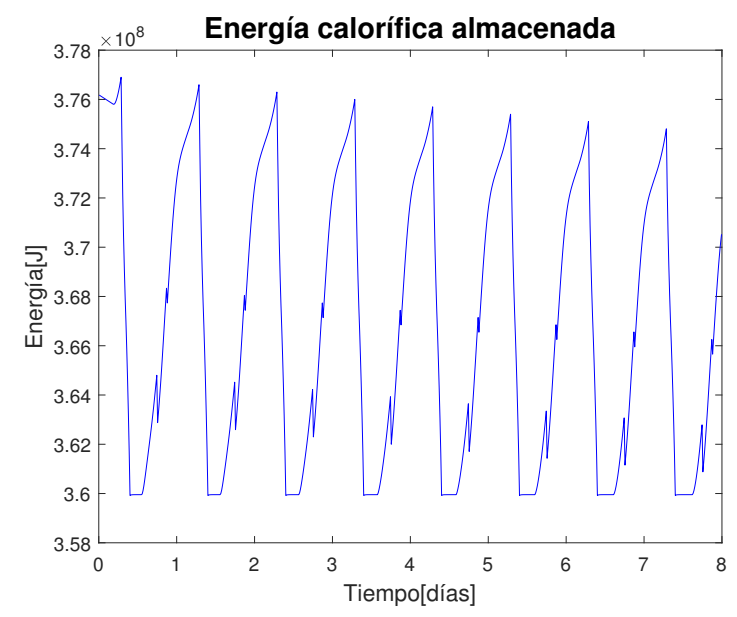

Figura 10: Energía calorífica almacenada en los depósitos a lo largo de ocho días del mes de abril

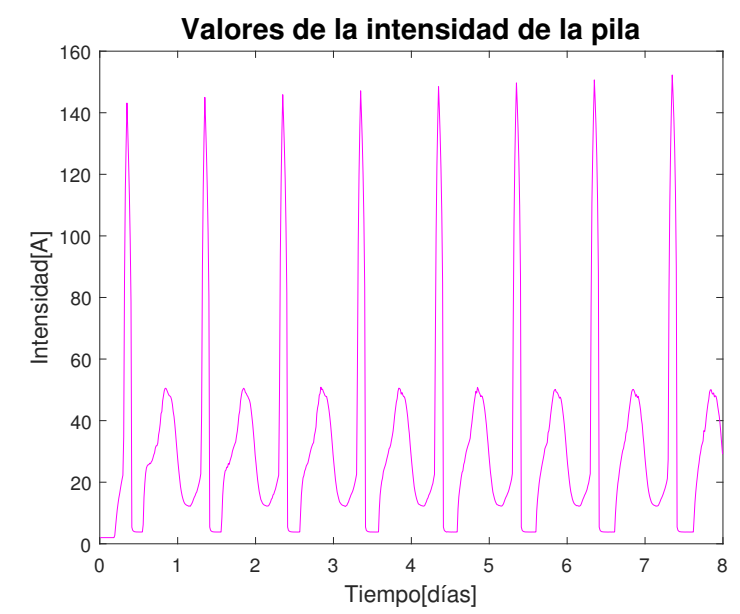

Figura 11: Corriente de la pila a lo largo de ocho días del mes de abril

\subsection{CONCLUSIONES}

En este artículo se ha presentado un sistema de gestión de energía para una casa alimentada con una pila de combustible tipo PEM de alta temperatura. Para ello se han definido las características de los depósitos de calefacción, ACS y batería eléctrica así como su modelo matemático simplificado. Dichos sistemas se han controlado a bajo nivel con controladores simples. Para el sistema de gestión de energía se ha planteado un problema de control predictivo basada en unas demandas previstas para una casa y un modelo del sistema general de depósitos para ajustar los valores producidos a todas horas de un día típico de abril a la demanda prevista. Finalmente, los resultados de las simulaciones se han presentado y comentado.

\section{Agradecimientos}

This work has been partially funded by the Spa- nish national project MICAPEM (ref. DPI201569286-C3-2-R, MINECO/FEDER). This work is supported by the Spanish State Research Agency through the María de Maeztu Seal of Excellence to IRI (MDM-2016-0656). This work is partially funded by AGAUR of Generalitat de Catalunya through the Advanced Control Systems (SAC) group grant (2017 SGR 482).

\section{English summary}

\section{HIGH TEMPERATURE PEM FUEL CELLS USAGE FOR COM- BINED HEAT AND POWER COM- FORT APPLICATIONS}

\section{Abstract}

Due to energy shortage and climate change effects world-wide, several alternatives to classical energy sources such as fossil fuels and nuclear fission power plants have arisen. In this context, combined heat and power appear as one of the possible solutions because of its ability to generate electrical and thermal energy more efficiently than conventional methods. Regarding this, high temperature proton exchange fuel cells enable the implementation of combined heat and power systems and have a lesser impact in the production of greenhouse effect gases to the one by conventional energy sources. In this article, an energy management system for a fuel-cellbased simple model controlled using predictive control is detailed. First of all, fuel cell characteristics as well as its contribution to the solution of the current energy problem are presented. Later on, modelling and low level control of the fuel cell and its heat and electricity generation subsystems are described and, finally, the proposed model predictive control problem is shown as a way to manage heat and electrical energy to fulfil a concrete residential demand.

Keywords: fuel cells, combined heat and power and model predictive control. 


\section{Referencias}

[1] H.R. Ellamla, I. Staffell, P. Bujlo, B.G. Pollet, S. Pasupathi, (2015) Current status of fuel cell based combined heat and power systems for residential sector, Journal of Power Sources, vol. 293, pp 312-328.

[2] D. Hissel, M.C. Pera, (2016) Diagnostic and

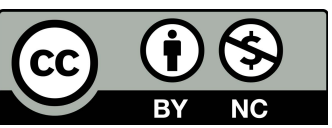

C 2018 by the authors. Submitted for possible open access publication under the terms and conditions of the Creative Commons Attribution CC-BY-NC 3.0 license (https://creativecommons.org/licenses/by-nc/3.0). health management of fuel cell systems: Issues and solutions, Annual Reviews in Control, vol. 42, pp 201-211.

[3] F. Barbir, (2012) PEM Fuel Cells: Theory and practice, 2nd Edition, Academic Press, Elsevier. 